\title{
Circular RNA hsa_circ_0078602 may have potential as a prognostic biomarker for patients with hepatocellular carcinoma
}

\author{
PEISI KOU ${ }^{1 *}$, CHENYUE ZHANG $^{2 *}$, JIAMAO LIN $^{3}$ and HAIYONG WANG ${ }^{3}$ \\ ${ }^{1}$ Department of Radiation Oncology, The First Affiliated Hospital of Zhengzhou University, Zhengzhou, Henan 45000; \\ ${ }^{2}$ Fudan University Shanghai Cancer Center, Shanghai 200032; ${ }^{3}$ Department of Internal Medicine-Oncology, \\ Shandong Cancer Hospital and Institute, Shandong Cancer Hospital Affiliated to Shandong University, \\ Shandong Academy of Medical Sciences, Jinan, Shandong 250117, P.R. China
}

Received February 26, 2018; Accepted September 17, 2018

DOI: $10.3892 / 01.2018 .9863$

\begin{abstract}
Circular RNA (circRNA), a type of endogenous non-coding RNA, is a closed continuous loop of RNA with no poly(A) tail. Previously, studies have identified that circRNAs are closely associated with several cancer types. However, their function in hepatocellular carcinoma (HCC) has rarely been studied. Therefore, the aim of the current study was to screen differential circRNA expression between HCC tissues and adjacent non-cancerous tissues, and test the potential clinical value of individual circRNAs. CircRNA microarray was used to investigate global circRNA expression profiles. Attention was then focused on the top four circRNAs whose expression levels were reduced in HCC tissues as compared with non-cancerous tissues. Additionally, RNA expression was validated in 30 matched tissue samples using reverse transcription-quantitative polymerase chain reaction. The results revealed that the expression levels of hsa_circ_0078602 and hsa_circ_0018764 were consistent with microarray analysis $(\mathrm{P}<0.05)$. Between these two circRNAs, hsa_circ_0078602 demonstrated an association with a favorable diagnostic efficiency, with an area under the receiver operating characteristic curve of $0.787(\mathrm{P}<0.001)$. To further verify the expression level of hsa_circ_0078602, the patient sample size was increased to 79. The results supported the conclusion that circ_0078602 was downregulated in HCC tissue compared with non-cancerous tissue $(\mathrm{P}=0.015)$ and exhibited diagnostic potential. Notably, it was identified that a lower hsa_circ_0078602 expression
\end{abstract}

Correspondence to: Dr Haiyong Wang, Department of Internal Medicine-Oncology, Shandong Cancer Hospital and Institute, Shandong Cancer Hospital Affiliated to Shandong University, Shandong Academy of Medical Sciences, 440 Jiyan Road, Jinan, Shandong 250117, P.R. China

E-mail: wanghaiyong6688@126.com

${ }^{*}$ Contributed equally

Key words: circular RNA, hsa_circ_0078602, hepatocellular carcinoma, biomarker, diagnosis, prognosis level was associated with a worse prognosis among patients with HCC. In addition, it was revealed that $9.0 \times 10^{-5}$ was the most efficient cut-off value of hsa_circ_0078602 expression to assess the outcomes of patients with HCC. The present study revealed that hsa_circ_0078602 may be a novel diagnostic biomarker of $\mathrm{HCC}$ and therefore have potential prognostic value.

\section{Introduction}

Circular RNAs (circRNAs) are a type of non-coding RNA that was first discovered by Sanger in a virus using electron microscopy in the 1970s (1). These molecules have a closed loop structure with no 5'-terminal cap or 3'-terminal poly(A) tail. This unique structure protects circRNAs from rapid degradation by exonucleases (2). CircRNAs have subsequently been identified in other organisms (3-6). Previously, these molecules were considered to be byproducts of aberrant RNA splicing and few studies have investigated circRNAs due to their low abundance and lack of known functions. Due to the rapid development of bioinformatics and RNA deep sequencing technology, an increased number of studies investigating circRNAs have been conducted in recent decades. CircRNAs are now thought to be endogenous, abundant, stable and conserved in mammalian cells (2), and they have been reported to have considerable biological function $(7,8)$.

CircRNAs perform their functions through various mechanisms. For instance, cerebellar-degeneration-related protein 1 antisense RNA (CDRlas) serves as competing endogenous RNA or a microRNA (miRNA) sponge to regulate gene expression by reducing the inhibitory effect of miRNA on its target gene (9). Additionally, previous studies have identified that circRNAs may regulate parental gene expression by binding to the RNA polymerase II transcription complex $(10,11)$. In addition, recent studies have demonstrated that circRNAs also bind with certain proteins (12) and DNA (13) to regulate downstream signaling. An increasing number of studies have revealed that circRNAs are involved in many diseases, including atherosclerosis (14), heart failure (15), Alzheimer's disease (16), diabetes (17), osteoarthritis (18) and cancer (19). Although 
a number of studies on cancer-associated circRNA have been performed (19-21), the function and clinical value of circRNAs in hepatocellular carcinoma (HCC) remain to be investigated.

The current study analyzed differential circRNA expression between HCC tissues and adjacent non-cancerous tissues, and validated the results of microarray analysis. Finally, hsa circ_0078602 was identified as having decreased expression in HCC tissues and being closely associated with the prognosis of patients with HCC. The current study also demonstrated the diagnostic potential of hsa_circ_0078602. Decreased expression of hsa_circ_0078602 in HCC tissue may act as a biomarker for diagnosis and prognosis prediction, and may also serve as a potential therapeutic target for HCC.

\section{Materials and methods}

Patients and tissue samples. HCC tissue samples and adjacent normal liver tissue samples were collected from 79 patients who underwent surgery at Fudan University Shanghai Cancer Center, between January 2011 and December 2015. The cohort consisted of $70(88.6 \%)$ males and $9(11.4 \%)$ females, with 56 (70.9\%) of the patients being $<60$ years old. All patients were pathologically diagnosed with $\mathrm{HCC}$ and provided written informed consent before sample collection. Patients with severe disorders of major organs or a history of any other tumors were excluded. Information on the serum $\alpha$-fetoprotein (AFP) levels, measured prior to surgery using ELISA, was obtained from the medical records of the patients. The overall survival (OS) time was calculated from the date of diagnosis to the date of mortality or the last known follow-up date. Following tissue procurement, the samples were immediately stored at $-80^{\circ} \mathrm{C}$ until further experiments. The present study was approved by the Ethics Committee of the Shandong Cancer Hospital Affiliated to Shandong University (Jinan, China).

RNA isolation and circRNA microarray analysis. Total RNA was extracted from HCC tissue and adjacent non-cancerous tissue using TRIzol reagent (Invitrogen; Thermo Fisher Scientific, Inc., Waltham, MA, USA) according to the manufacturer's protocol and RNA integrity was determined with agarose gel electrophoresis (1\% gel). Qualified RNA was used for microarray detection or subsequent validation experiments. CapitalBio Corporation (Beijing, China) performed the circRNA microarray detection, with 6 samples (3 HCC and 3 paired non-cancerous samples). The CapitalBio Human CircRNA Array v2 was designed with 4 identical arrays per slide (4x180,000 format), with each array containing probes interrogating $\sim 170,340$ human circRNAs, selected based on known circRNA sites included in CircBase (http://www.circbase.org/) and deepBase (http://rna.sysu.edu. $\mathrm{cn} /$ deepBase/). The circRNA array data was analyzed using GeneSpring software (version 13.0; Agilent Technologies, Inc., Santa Clara, CA, USA). Threshold values of $\geq 2$ and $\leq-2$-fold change, and $\mathrm{P}<0.05$ were used to select the differentially expressed genes.

Reverse transcription-quantitative polymerase chain reaction (RT-qPCR). Complementary DNA was synthesized by RT using the RNA-to-cDNA kit (Takara Bio, Inc., Otsu, Japan), according to the manufacturer's protocol. The relative expression of circRNA was determined using the ABI Prism 7900 Sequence Detection system (Applied Biosystems; Thermo Fisher Scientific, Inc.). The qPCR assay was conducted using a SYBR Green kit (Takara Bio Inc., Otsu, Japan), with the following thermocycling conditions: 45 cycles consisting of denaturation at $95^{\circ} \mathrm{C}$ for $5 \mathrm{sec}$, annealing at $60^{\circ} \mathrm{C}$ for $30 \mathrm{sec}$ and extension at $72^{\circ} \mathrm{C}$ for $30 \mathrm{sec}$. A housekeeping gene, GAPDH, was used as a control and RNA enrichment was analyzed using the $2^{-\triangle \Delta C q}$ method (22). All primers were synthesized by ThinkGene Biotech Co., Ltd. (Shanghai, China). The primer sequences are listed in Table I.

Statistical analysis. All statistical analyses were performed using SPSS software (version 19.0; IBM Corp., Armonk, NY, USA) and GraphPad Prism software (version 5.0; GraphPad Software, Inc., La Jolla, CA, USA). Differences in circRNA expression between HCC and paired non-cancerous tissues were assessed using the paired t-test. A receiver operating characteristic (ROC) curve and the area under the curve (AUC) was used to assess diagnostic power. Survival curves were obtained using the Kaplan-Meier method and the differences between the curves were analyzed using a log rank test. X-tile (version 3.5.0 software; https://medicine.yale. $\mathrm{edu} / \mathrm{lab} / \mathrm{rimm} / \mathrm{research} /$ software.aspx) was used to analyze the optimal cut-off value of hsa_circ_0078602 expression. $\mathrm{P} \leq 0.05$ was considered to indicate a statistically significant difference.

\section{Results}

Dysregulated circRNAs in HCC tissues relative to non-cancerous tissues. To explore differentially expressed circRNAs in HCC tumor tissue and adjacent non-tumor liver tissue, tissue samples from 3 patients were selected to perform circRNA microarray detection. Downregulated circRNAs in HCC tissue were of most interest, therefore the 20 circRNAs with the most suppressed expression in HCC tissues are presented in Fig. 1A. As demonstrated in Fig. 1B, the expression levels of global genes in the probe in different samples after standardization exhibited similar levels. Principal component analysis was also conducted to reflect the similarity of samples (Fig. 1C).

Validation of circRNA microarray results. Among circRNAs identified to be present at decreased levels in HCC tissue compared with non-cancerous tissue, the four most suppressed examples (hsa_circ_0078602, hsa_circ_0094117, hsa_circ_0127245 and hsa_circ_0018764) were selected for further verification experiments. The expression of these four circRNAs in HCC and paired non-cancerous tissues from 30 patients was analyzed by RT-qPCR (Fig. 2). It was identified that the expression of hsa_circ_0078602 and hsa_circ_0018764 were significantly and consistently decreased in HCC tissues compared with matched samples (Fig. 2A and D). However, there was no significant difference in the expression of hsa_circ_0094117 between the tumor and the healthy tissues (Fig. 2B). Furthermore, the expression of hsa_circ_0127245 was increased in the tumor tissue compared with the adjacent tissue, in contradiction to the microarray result (Fig. 2C). As the reason for this inconsistency required 
Table I. Primer sequences for reverse transcription-quantitative polymerase chain reaction analysis.

\section{CircRNA name}

hsa_circ_0078602

hsa_circ_0094117

hsa_circ_0127245

hsa_circ_0018764

GAPDH
Primer sequences

Forward 5'-TGGCCATGTCAAATTTGTTG-3'

Reverse 5'-CATGTAGTTGGGCGAGAAGG-3'

Forward 5'-CTCCTACCGCTGTGAGTGTG-3'

Reverse 5'-TCAAAGCATGTCTGCCTGTC-3'

Forward5'-TGATTTTTGTTCCATCGTATATCAA-3'

Reverse 5'-TTCATCCTTGGTGCTGAGAA-3'

Forward 5'-TGGAGCTCTTCCTGACCAAC-3'

Reverse 5'-CACCGTTGTTTTGCTCACAT-3'

Forward 5'-TCGACAGTCAGCCGCATCTTCTTT-3'

Reverse 5'-ACCAAATCCGTTGACTCCGACCTT-3'

CircRNA, circular RNA.

A

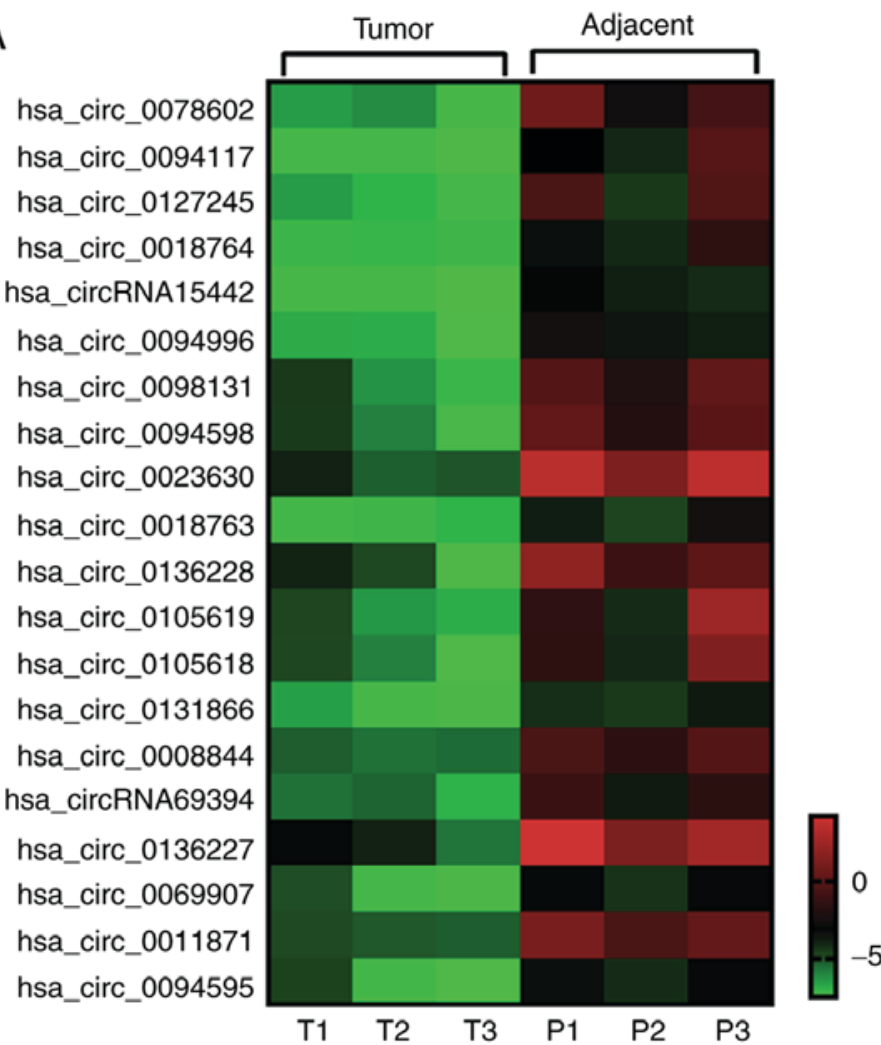

B
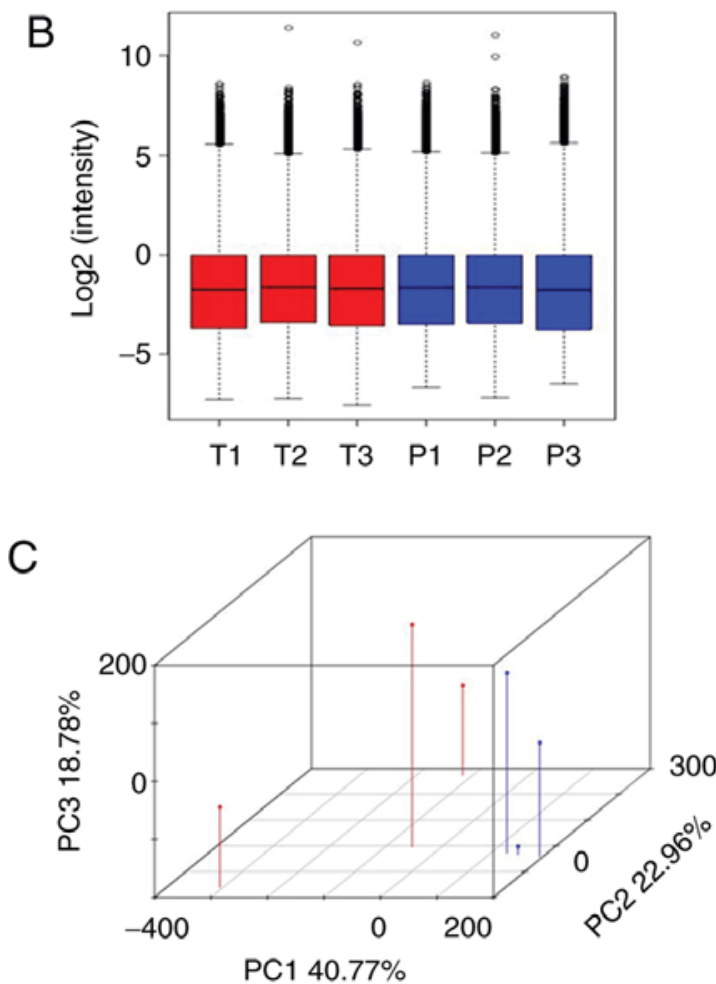

Figure 1. Downregulated circRNAs in HCC tissues. (A) Based on circRNA microarray results, a heat map of the 20 most decreased circRNAs in hepatocellular carcinoma tissues relative to paired non-cancerous tissues was constructed. Green color represents low expression and red color represents high expression. (B) A boxplot was used to compare the expression levels of global genes in the probe for different samples after normalization. (C) Principal component analysis reflected the similarity of the samples. circRNA, circular RNA; T, tumor tissue; P, paired non-cancerous tissue; PC, principal component.

clarification with further testing, hsa_circ_0127245 was not used in subsequent experiments. Next, the clinical role of hsa_circ_0078602 and hsa_circ_0018764 in patients with HCC was investigated. The potential diagnostic effect of hsa_circ_0078602 and hsa_circ_0018764 in distinguishing $\mathrm{HCC}$ tumor tissues from neighboring non-cancerous tissues was examined by establishing an ROC curve (Fig. 3). The AUCs for hsa_circ_0078602 and hsa_circ_0018764 were 0.787 and $0.67 \overline{6}$, respectively, indicating that the former may have potential to be a diagnostic biomarker. Therefore, attention was focused on hsa_circ_0078602 for further study.

Next, the expression of hsa_circ_0078602 in HCC tumor tissues was confirmed in 79 patients with HCC; it was revealed to be significantly decreased in HCC tissues compared with adjacent non-cancerous tissues (Fig. 4A). In addition, hsa_circ_0078602 demonstrated good diagnostic efficacy (Fig. 4B). Although the AUC in Fig. 4B is $0.587, \mathrm{P}<0.05$ indicated statistical significance, indicating that hsa-circ-0078602 

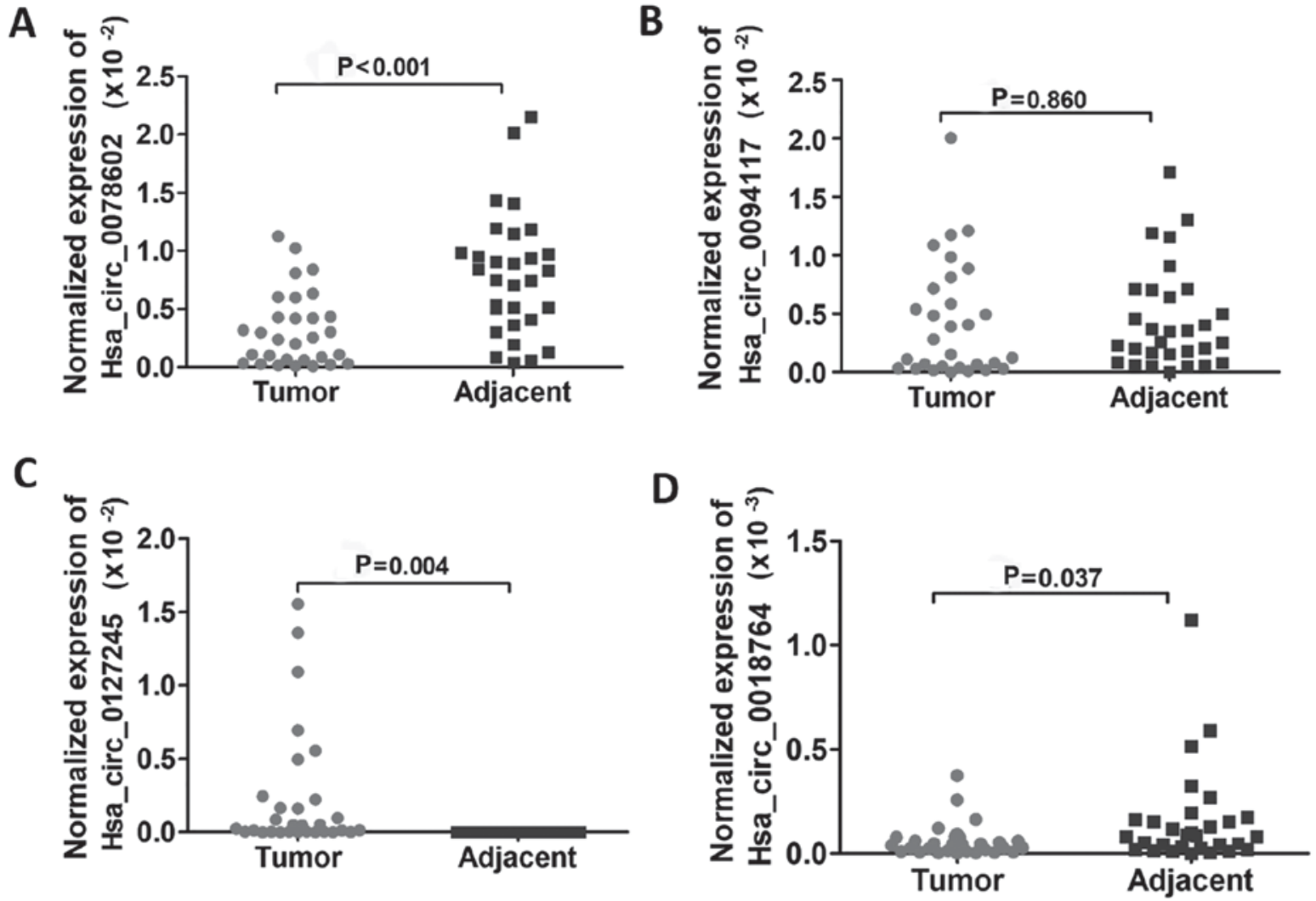

Figure 2.Validation of candidate circular RNAs in 30 HCC patient samples using quantitative polymerase chain reaction.(A) The expression of hsa_circ_0078602 was significantly decreased in HCC tissues compared with adjacent non-cancerous samples $(\mathrm{P}<0.001)$. (B) No significant difference was identified in the expression of hsa_circ_0094117 between HCC tissues and adjacent non-cancerous tissues ( $\mathrm{P}=0.860)$. (C) The expression of hsa_circ_0127245 was relatively high in HCC tissues, which was inconsistent with the result of microarray. (D) The expression of hsa_circ_0018764 was significantly decreased in HCC tissues compared with adjacent non-cancerous samples $(\mathrm{P}=0.037)$. This result is in accordance with microarray analysis. HCC, hepatocellular carcinoma.

may have potential as a diagnostic biomarker. The clinicopathological characteristics of the 79 patients and the expression levels of hsa_circ_0078602 are presented in Table II.

Low hsa_circ_0078602 expression is associated with poor survival outcome in patients with HCC. Since the expression level of hsa_circ_0078602 was significantly different between HCC tumor tissue and neighboring non-tumor tissue, the association of hsa_circ_0078602 expression with the survival of patients with HCC was investigated. Analysis was performed to assess the association between expression of hsa_circ_0078602 and overall survival of patients with HCC in 79 cases. Kaplan-Meier survival analysis was used and patients were divided into high and low hsa_circ_0078602 expression groups ( $\mathrm{P}=0.035$; Fig. 5A). The data suggested that patients with low hsa_circ_0078602 expression had significantly poorer survival outcomes. These analyses demonstrated that hsa_circ_0078602 expression level may have potential to predict the prognosis of patients with HCC. Next, the association between expression level of hsa_circ_0078602 and the clinical parameter, serum AFP was analyzed. Patients were stratified into two cohorts based on their level of serum AFP ( $<200$ and $\geq 200 \mathrm{ng} / \mathrm{ml})$. The difference of overall survival between high and low hsa_circ_0078602 expression groups within the two cohorts was investigated (Fig. 5B and C). No statistically significant
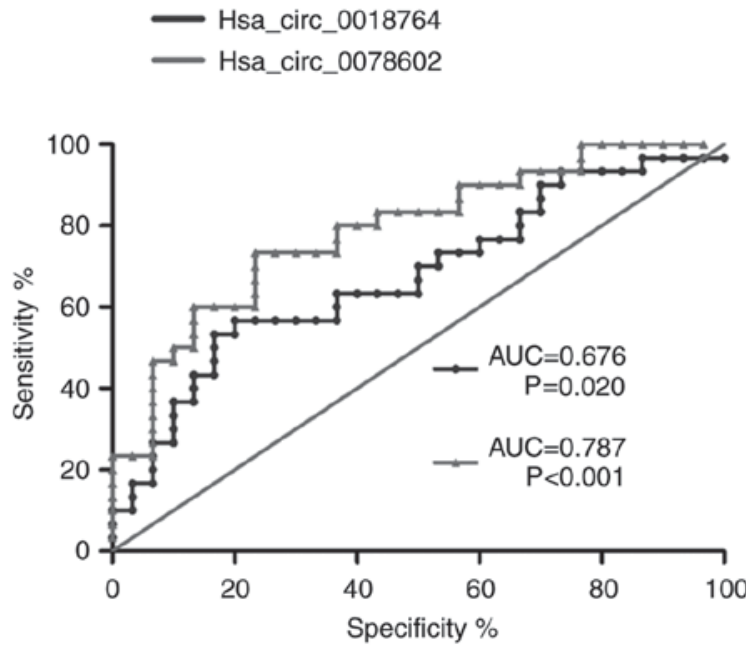

Figure 3. Diagnostic potential of hsa_circ_0078602 in hepatocellular carcinoma. Receiver operating characteristic curve analysis of hsa_circ_0078602 and hsa_circ_0018764 suggested hsa_circ_0078602 has a higher ability to discriminate between tumor and non-cancerous samples (AUC=0.787; $\mathrm{P}<0.001)$ compared with hsa_circ_0018764 (AUC=0.676, $\mathrm{P}=0.20)$. AUC, area under curve.

difference was identified between the high and low expression groups in the $<200 \mathrm{ng} / \mathrm{ml}(\mathrm{P}=0.146)$, or the $\geq 200 \mathrm{ng} / \mathrm{ml}$ AFP cohort $(\mathrm{P}=0.309)$. 
A

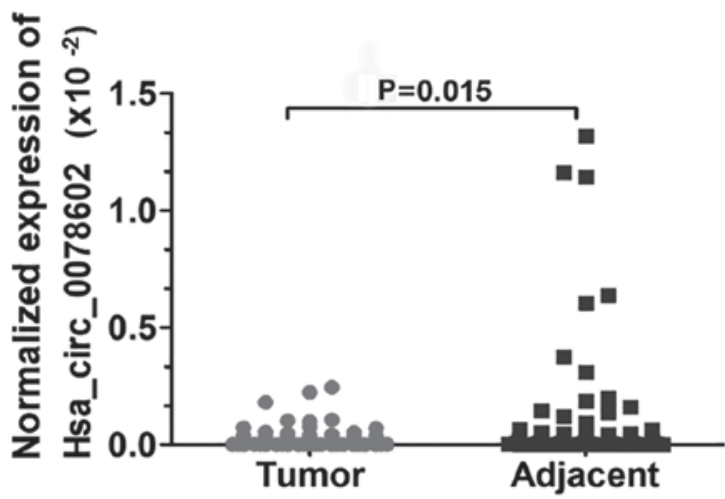

B

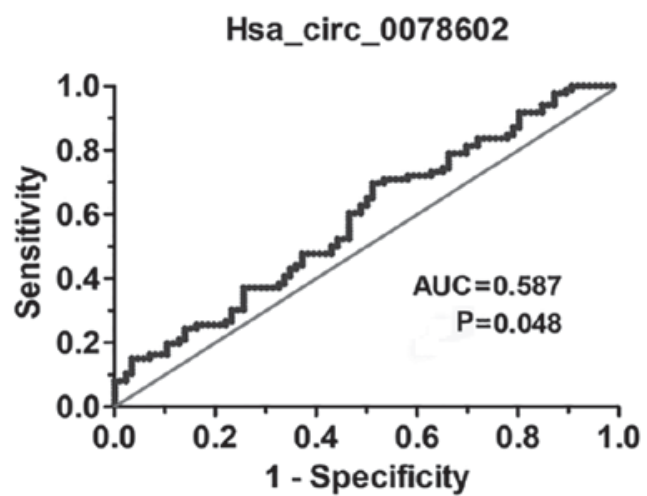

Figure 4. Further validation of hsa_circ_0078602. (A) Reverse transcription-quantitative polymerase chain reaction with 79 samples demonstrated lower hsa circ_0078602 expression in HCC tumor tissues compared with non-cancerous tissues. (B) Receiver operating characteristic curve analysis of hsa_circ_0078602 revealed its potential as a diagnostic marker for $\mathrm{HCC}(\mathrm{AUC}=0.587 ; \mathrm{P}=0.048$ ). HCC, hepatocellular carcinoma; AUC, area under curve.

Table II. Clinicopathological characteristics of the patients with hepatocellular carcinoma $(n=79)$ and relative expression levels of hsa_circ_0078602.

\begin{tabular}{|c|c|c|}
\hline Variable & $\begin{array}{l}\text { Patient number } \\
\qquad(\%)\end{array}$ & $\begin{array}{c}\text { Mean } \pm \text { SD } \\
\left(\times 10^{-4}\right)\end{array}$ \\
\hline \multicolumn{3}{|l|}{ Sex } \\
\hline Male & $70(88.6)$ & $5.55 \pm 19.65$ \\
\hline Female & $9(11.4)$ & $6.02 \pm 9.16$ \\
\hline \multicolumn{3}{|l|}{ Age, years } \\
\hline$<60$ & $56(70.9)$ & $7.24 \pm 22.03$ \\
\hline$\geq 60$ & $23(29.1)$ & $1.62 \pm 2.07$ \\
\hline \multicolumn{3}{|l|}{ Cirrhosis } \\
\hline Positive & $30(38.0)$ & $3.68 \pm 11.89$ \\
\hline Negative & $49(62.0)$ & $6.78 \pm 21.84$ \\
\hline \multicolumn{3}{|c|}{ Serum AFP, ng/ml } \\
\hline$<200$ & $44(55.7)$ & $7.20 \pm 2.43$ \\
\hline$\geq 200$ & $35(44.3)$ & $3.60 \pm 6.84$ \\
\hline \multicolumn{3}{|c|}{ Vascular cancer embolus } \\
\hline Positive & $48(60.8)$ & $3.03 \pm 6.11$ \\
\hline Negative & $31(39.2)$ & $9.59 \pm 28.51$ \\
\hline \multicolumn{3}{|c|}{ Intrahepatic metastasis } \\
\hline Positive & $23(29.1)$ & $4.58 \pm 8.16$ \\
\hline Negative & $56(70.9)$ & $6.02 \pm 21.64$ \\
\hline \multicolumn{3}{|c|}{ Extrahepatic metastasis } \\
\hline Positive & $25(31.6)$ & $4.44 \pm 7.84$ \\
\hline Negative & $54(68.4)$ & $6.14 \pm 22.03$ \\
\hline
\end{tabular}

AFP, $\alpha$-fetoprotein; SD, standard deviation.

Analysis of optimum cut-off value of hsa_circ_0078602 expression. Finally, X-tile 3.5.0 software was used to calculate the optimal cut-off value of hsa_circ_0078602 expression, which could most robustly estimate outcomes of patients with
HCC (Fig. 6). The data demonstrated that an hsa_circ_0078602 relative expression of $9.0 \times 10^{-5}$ (normalized to housekeeping gene GAPDH) may be effective in predicting survival outcomes of patients with HCC. Patients with hsa_circ_0078602 relative expression levels $<9.0 \times 10^{-5}$ may exhibit a poor prognosis.

\section{Discussion}

On a global scale, primary liver cancer is a major contributor to both cancer incidence and cancer-associated cases of mortality (23). Liver cancer is the third most common type of cancer diagnosed in China (24) and is one of the leading causes of cancer-associated cases of mortality worldwide (25). $\mathrm{HCC}$, the most common type of primary liver cancer, accounts for approximately 80 to $85 \%$ of primary liver cancer cases worldwide (26). With advancements in diagnostic and therapeutic approaches, certain patients with HCC could be diagnosed at an early stage and undergo radical surgery. Nonetheless, the prognosis of most patients with HCC remains unsatisfactory, mainly due to metastasis, recurrence or drug resistance $(27,28)$. These clinical challenges promote studies to identify new biomarkers to diagnose HCC and reveal novel targets for the development of more effective therapies.

Currently, the roles of circRNAs in cancer have received widespread attention from researchers. For example, circRNAs have been revealed to be dysregulated in certain tumor types, including breast cancer (29), lung cancer (30), esophageal squamous cell carcinoma (31), gastric cancer (32) and colorectal carcinoma (33), and these circRNAs have been identified to be closely associated with tumorigenesis and tumor progression. However, to the best of our knowledge, the function of circRNAs in HCC is unknown. Rapid advancements in microarray analysis technology have promoted its widespread application in the study of non-coding RNA. The current study analyzed differential expression of circRNA between HCC tissues and adjacent non-cancerous tissues in three paired HCC samples using microarray analysis.

The results demonstrated that circRNA levels differed between $\mathrm{HCC}$ tissues and the adjacent normal tissues. Both 

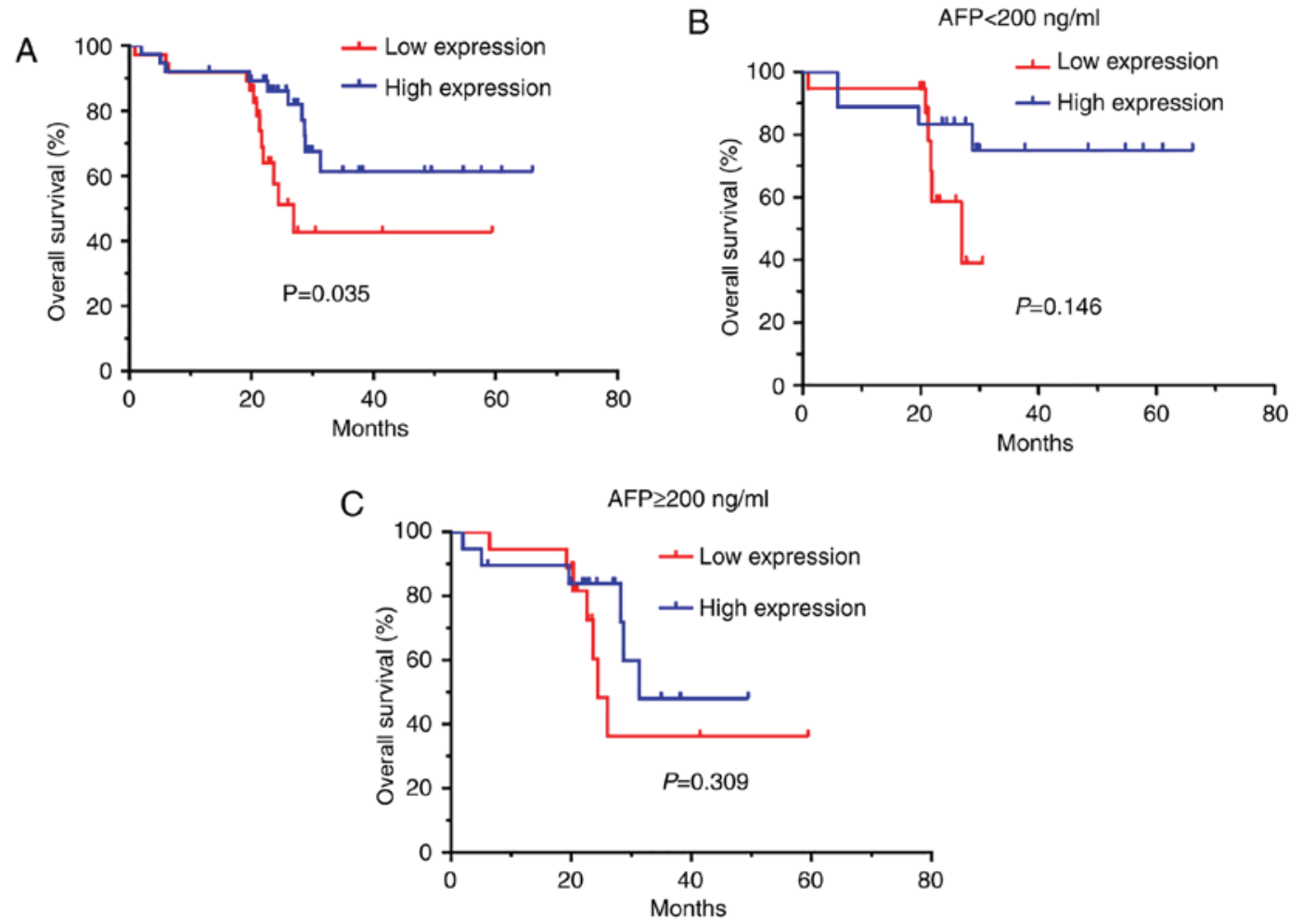

Figure 5. Ability of hsa_circ_0078602 in predicting survival outcome of patients with HCC and the association with serum AFP. (A) Kaplan-Meier survival analysis was performed to measure the association between hsa_circ_0078602 and OS of patients with HCC. The result identified that worse prognosis was associated with lower hsa_circ_0078602 expression $(\mathrm{P}=0.035)$. (B and C) Patients were divided into two cohorts according to the level of serum AFP. It was identified that there was no association between OS and the expression level of hsa_circ_0078602 in either the high or low AFP cohort. AFP, $\alpha$-fetoprotein; OS, overall survival; HCC, hepatocellular carcinoma.

A

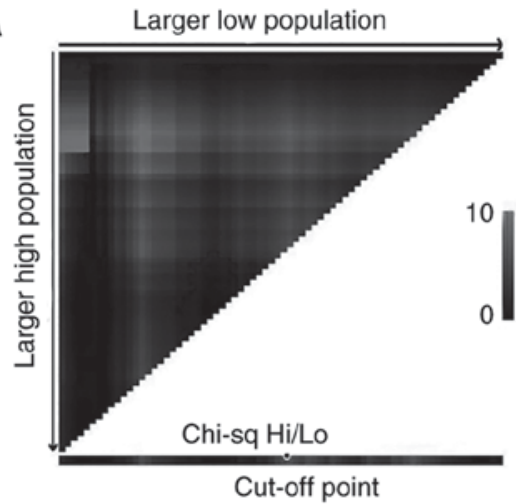

C

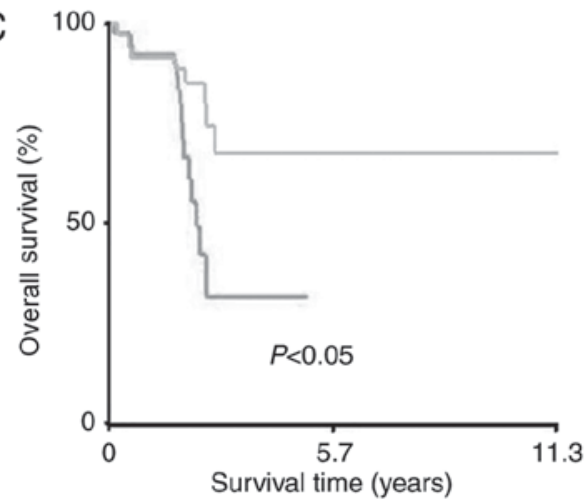

B

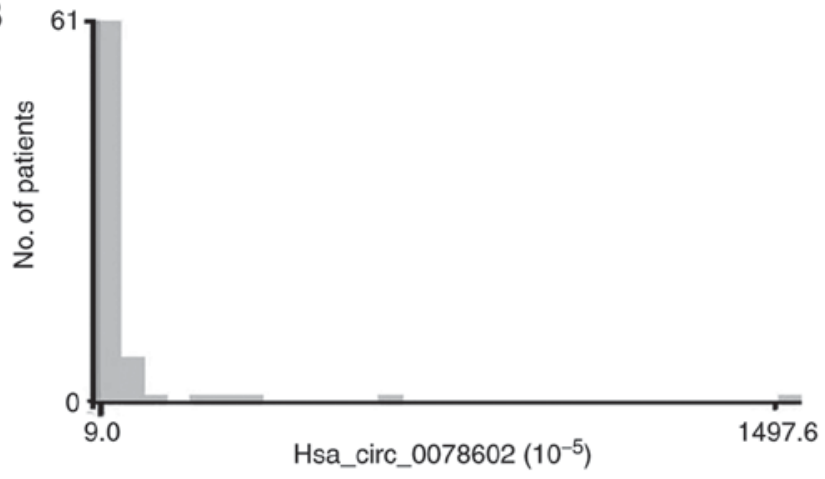

D

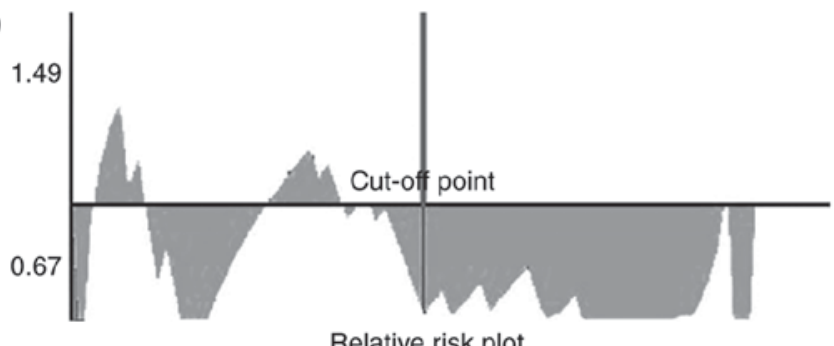

Figure 6. Calculation of the optimal cut-off value for hsa_circ_0078602 expression. (A) X-tile plot was used to analyze the efficient cut-off value. (B) Histogram plot demonstrated that $9.0 \times 10^{-5}$ was the optimal cut-off value to predict outcomes for patients with HCC. (C) Survival curve for patients with HCC, stratified based on the critical value, suggested that the survival difference was statistically significant $(\mathrm{P}<0.05)$. (D) Continuous relative risk plot revealed that patients with hsa_circ_0078602 expression below the cut-off point may have worse prognosis. HCC, hepatocellular carcinoma. 
upregulated and downregulated circRNAs were identified in HCC tissues compared with adjacent normal tissues. Following a relevant study that demonstrated that certain downregulated circRNAs may serve a role in hepatocellular carcinoma progression (34), the current study was more focused on downregulated circRNAs. To validate the results of microarray, RNA expression levels of the four most downregulated circRNAs were measured in a total of 30 matched HCC samples. As indicated by RT-qPCR, the levels of hsa_circ_0078602 and hsa_circ_0018764 were consistent with microarray analysis. Previous studies have suggested a potential role of circRNA as a biomarker in cancer diagnosis $(35,36)$. Therefore, the current study explored the clinical value of circRNAs. Notably, the AUC of hsa_circ_0078602 was 0.787 , demonstrating a favorable diagnostic efficiency, suggesting that further studies should be performed. The level of hsa_circ_0078602 was subsequently confirmed in 79 paired HCC tissues. The results demonstrated that the expression level of hsa_circ_0078602 in HCC tissue was significantly lower compared with non-cancerous tissue. These data suggest that hsa_circ_0078602 may be used as a novel biomarker that can contribute to the identification of HCC.

Next, it was identified that the decreased hsa_circ_0078602 expression in HCC tissue was significantly associated with a worse prognosis. The expression level of hsa_circ_0078602 in HCC tissues was significantly lower compared with adjacent non-cancerous tissues, which indicated that hsa_circ_0078602 acted as a tumor suppressor gene for carcinogenesis of HCC; the results of Kaplan-Meier analysis was consistent with this function. Considering the role of serum AFP in HCC diagnosis and efficacy evaluation, the current study investigated whether the association between circRNA and OS was affected by the level of serum AFP. However, no significant difference was identified in OS when patients were divided into two cohorts according to the level of serum AFP. It was speculated that there may be some uncertain connection between hsa_circ_0078602 and serum AFP, which requires further study. Finally, an optimal cut-off value of hsa_circ_0078602 $\left(9.0 \times 10^{-5}\right)$ for the prognostic prediction was obtained using X-tile 3.5.0 software. Although the application of a circRNA cut-off value still needs to be confirmed in future studies, it is assumed that the hsa_circ_0078602 expression value of $9.0 \times 10^{-5}$ may provide value in predicting the outcomes of patients with HCC.

To date, few studies have focused on the association between HCC and circRNA, particularly regarding function and possible mechanism. Yang et al (37) reported that CDR1as exerted an effect on the proliferation of HCC cells partly through regulation of epidermal growth factor receptor signaling via control of miR-7 expression, which provided a reliable and highly efficient method for globally identifying circRNA-regulated proteins. Using RNA-sequencing, Zheng et al (38) identified that a circRNA derived from exon 2 of homeodomain interacting protein kinase 3, termed circHIPK3, was differentially expressed in $\mathrm{HCC}$ and regulated human cell growth by directly binding to miR-124 and inhibiting miR-124 activity. Similarly, Han et al (34) revealed that mitochondrial translation optimization 1 homologue (circMTO1) expression was significantly decreased in HCC and associated with poor prognosis of patients with HCC. In addition, circMTO1 inhibited HCC growth via sponge activity on miR-9 and upregulation of p21 expression. These studies indicated that circRNA could regulate gene expression by serving as an miRNA sponge, which was confirmed in other previous studies $(9,39)$. The circRNA identified in the current study (hsa_circ_0078602) was identified to be associated with prognosis of patients with HCC. Following a bioinformatics analysis to explore possible mechanisms, it was revealed that hsa_circ_0078602 is associated with several miRNAs, including hsa-miR-1207-5p, hsa-miR-6787-5p, hsa-miR-940, hsa-miR-1202, hsa-miR-4459 and hsa-miR-3194-5p, which have been identified to affect cell proliferation, cell cycle progression and apoptosis in tumor cell lines. The present study hypothesizes that hsa_circ_0078602 may affect the function of tumor cells via miRNA. The association between hsa_circ_0078602 and miRNA may be revealed and validated in further studies.

In conclusion, the current data demonstrated that hsa_circ_0078602 expression was significantly decreased in HCC and may be utilized as a circRNA biomarker for the diagnosis of HCC. Survival analysis demonstrated that lower expression of hsa_circ_0078602 in HCC tissue was associated with a worse prognosis. Notably, it was also demonstrated that an hsa_circ_0078602 relative expression level $<9.0 \times 10^{-5}$ may indicate a poor prognosis.

\section{Acknowledgements}

Not applicable.

\section{Funding}

This study was supported jointly by the National Natural Science Foundation of China (no. 81603348), China Postdoctoral Fund (no. 21300075311104), Shandong Postdoctoral Innovation Special Fund (no. 201602012), China Postdoctoral Special Fund (no. 2018T110696), Shandong Province Key R \& D Plan (no. 2018GSF119014) and the CSCO - Lizhu Chinese Medicine Cancer Research Fund (no. Y-L2018-009).

\section{Availability of data and materials}

All data generated or analyzed during the present study are included in this published article.

\section{Authors' contributions}

PK performed the experiments, analysis and was a major contributor in writing the manuscript. $\mathrm{CZ}$ also performed some experiments and collected the clinical information of all patients. JL made substantial amendments to the manuscript and participated in the data analysis. HW made significant contributions to conception, design and data analysis, and provided final approval for the study to be published. All authors read and approved the final manuscript.

\section{Ethics approval and consent to participate}

The present study was approved by the Ethics Committee of the Shandong Cancer Hospital Affiliated to Shandong University. 


\section{Patient consent for publication}

Not applicable.

\section{Competing interests}

The authors declare that they have no competing interests.

\section{References}

1. Sanger HL, Klotz G, Riesner D, Gross HJ and Kleinschmidt AK Viroids are single-stranded covalently closed circular RNA molecules existing as highly base-paired rod-like structures. Proc Natl Acad Sci USA 73: 3852-3856, 1976.

2. Jeck WR, Sorrentino JA, Wang K, Slevin MK, Burd CE, Liu J, Marzluff WF and Sharpless NE: Circular RNAs are abundant, conserved, and associated with ALU repeats. RNA 19: 141-157, 2013.

3. Hsu MT and Coca-Prados M: Electron microscopic evidence for the circular form of RNA in the cytoplasm of eukaryotic cells. Nature 280: 339-340, 1979.

4. Matsumoto Y, Fishel R and Wickner RB: Circular single-stranded RNA replicon in Saccharomyces cerevisiae. Proc Natl Acad Sci USA 87: 7628-7632, 1990.

5. Capel B, Swain A, Nicolis S, Hacker A, Walter M, Koopman P, Goodfellow P and Lovell-Badge R: Circular transcripts of the testis-determining gene Sry in adult mouse testis. Cell 73: 1019-1030, 1993

6. Danan M,Schwartz S,Edelheit S and SorekR: Transcriptome-wide discovery of circular RNAs in Archaea. Nucleic Acids Res 40: 3131-3142, 2012.

7. Memczak S, Jens M, Elefsinioti A, Torti F, Krueger J, Rybak A, Maier L, Mackowiak SD, Gregersen LH, Munschauer M, et al Circular RNAs are a large class of animal RNAs with regulatory potency. Nature 495: 333-338, 2013.

8. Guo JU, Agarwal V, Guo H and Bartel DP: Expanded identification and characterization of mammalian circular RNAs. Genome Biol 15: 409, 2014

9. Hansen TB, Jensen TI, Clausen BH, Bramsen JB, Finsen B, Damgaard CK and Kjems J: Natural RNA circles function as efficient microRNA sponges. Nature 495: 384-388, 2013.

10. Zhang Y, Zhang XO, Chen T, Xiang JF, Yin QF, Xing YH, Zhu S, Yang L and Chen LL: Circular intronic long noncoding RNAs. Mol Cell 51: 792-806, 2013.

11. Li Z, Huang C, Bao C, Chen L, Lin M, Wang X, Zhong G, Yu B, $\mathrm{Hu}$ W, Dai L, et al: Exon-intron circular RNAs regulate transcription in the nucleus. Nat Struct Mol Biol 22: 256-264, 2015.

12. Du WW, Yang W, Liu E, Yang Z, Dhaliwal P and Yang BB: Foxo3 circular RNA retards cell cycle progression via forming ternary complexes with $\mathrm{p} 21$ and CDK2. Nucleic Acids Res 44: 2846-2858, 2016.

13. Conn VM, Hugouvieux V, Nayak A, Conos SA, Capovilla G, Cildir G, Jourdain A, Tergaonkar V, Schmid M, Zubieta C and Conn SJ: A circRNA from SEPALLATA3 regulates splicing of its cognate mRNA through R-loop formation. Nat Plants 3 : 17053, 2017.

14. Burd CE, Jeck WR, Liu Y, Sanoff HK, Wang Z and Sharpless NE: Expression of linear and novel circular forms of an INK4/ARF-associated non-coding RNA correlates with atherosclerosis risk. PLoS Genet 6: e1001233, 2010.

15. Wang K, Long B, Liu F, Wang JX, Liu CY, Zhao B, Zhou LY, Sun T, Wang M, Yu T, et al: A circular RNA protects the heart from pathological hypertrophy and heart failure by targeting miR-223. Eur Heart J 37: 2602-2611, 2016.

16. Zhao Y, Alexandrov PN, Jaber V and Lukiw WJ: Deficiency in the ubiquitin conjugating enzyme UBE2A in Alzheimer's Disease (AD) is linked to deficits in a natural circular miRNA-7 sponge (circRNA; ciRS-7). Genes (Basel) 7: pii: E116, 2016.

17. $\mathrm{Xu} \mathrm{H}$, Guo S, Li W and Yu P: The circular RNA Cdrlas, via miR-7 and its targets, regulates insulin transcription and secretion in islet cells. Sci Rep 5: 12453, 2015.

18. Liu Q, Zhang X, Hu X, Dai L, Fu X, Zhang J and Ao Y: Circular RNA related to the chondrocyte ECM regulates MMP13 expression by functioning as a MiR-136 'Sponge' in human cartilage degradation. Sci Rep 6: 22572, 2016.
19. Hansen TB, Kjems J and Damgaard CK: Circular RNA and miR-7 in cancer. Cancer Res 73: 5609-5612, 2013.

20. Li Y, Dong Y, Huang Z, Kuang Q, Wu Y, Li Y and Li M Computational identifying and characterizing circular RNAs and their associated genes in hepatocellular carcinoma. PLoS One 12: e0174436, 2017.

21. Begum S, Yiu A, Stebbing J and Castellano L: Novel tumour suppressive protein encoded by circular RNA, circ-SHPRH, in glioblastomas. Oncogene 37: 4055-4057, 2018.

22. Livak KJ and Schmittgen TD: Analysis of relative gene expression data using real-time quantitative PCR and the 2(-Delta Delta C(T)) method. Methods 25: 402-408, 2001

23. McGlynn KA and London WT: The global epidemiology of hepatocellular carcinoma: Present and future. Clin Liver Dis 15: 223-243, vii-x, 2011

24. Chen W, Zheng R, Baade PD, Zhang S, Zeng H, Bray F, Jemal A, Yu XQ and He J: Cancer statistics in China, 2015. CA Cancer J Clin 66: 115-132, 2016.

25. Torre LA, Bray F, Siegel RL, Ferlay J, Lortet-Tieulent J and Jemal A: Global cancer statistics, 2012. CA Cancer J Clin 65: 87-108, 2015.

26. Gelband H, Chen CJ, Chen W, Franceschi S, Hall SA, London WT, McGlynn KA and Wild CP: Liver Cancer. In: Cancer: Disease control priorities, third edition (Volume 3). Gelband H, Jha P, Sankaranarayanan R and Horton S (eds.), The International Bank for Reconstruction and Development/The World Bank, Washington (DC), 2015.

27. Sasaki Y, Yamada T, Tanaka H, Ohigashi H, Eguchi H, Yano M, Ishikawa $\mathrm{O}$ and Imaoka $\mathrm{S}$ : Risk of recurrence in a long-term follow-up after surgery in 417 patients with hepatitis B- or hepatitis C-related hepatocellular carcinoma. Ann Surg 244: 771-780, 2006

28. Bruix J, Gores GJ and Mazzaferro V: Hepatocellular carcinoma: Clinical frontiers and perspectives. Gut 63: 844-855, 2014.

29. Lu WY: Roles of the circular RNA circ-Foxo3 in breast cancer progression. Cell Cycle 16: 589-590, 2017.

30. Zhu X, Wang X, Wei S, Chen Y, Chen Y, Fan X, Han S and Wu G: hsa_circ_0013958: A circular RNA and potential novel biomarker for lung adenocarcinoma. FEBS J 284: 2170-2182, 2017.

31. Su H, Lin F, Deng X, Shen L, Fang Y, Fei Z, Zhao L, Zhang X, Pan H, Xie D, et al: Profiling and bioinformatics analyses reveal differential circular RNA expression in radioresistant esophageal cancer cells. J Transl Med 14: 225, 2016.

32. Chen J, Li Y, Zheng Q, Bao C, He J, Chen B, Lyu D, Zheng B, $\mathrm{Xu} Y$, Long Z, et al: Circular RNA profile identifies circPVT1 as a proliferative factor and prognostic marker in gastric cancer. Cancer Lett 388: 208-219, 2017.

33. Hsiao KY, Lin YC, Gupta SK, Chang N, Yen L, Sun HS and Tsai SJ: Noncoding effects of circular RNA CCDC66 promote colon cancer growth and metastasis. Cancer Res 77: 2339-2350, 2017.

34. Han D, Li J, Wang H, Su X, Hou J, Gu Y, Qian C, Lin Y, Liu X, Huang M, et al: Circular RNA circMTO1 acts as the sponge of microRNA-9 to suppress hepatocellular carcinoma progression. Hepatology 66: 1151-1164, 2017.

35. Li Y, Zheng Q, Bao C, Li S, Guo W, Zhao J, Chen D, Gu J, He X and Huang S: Circular RNA is enriched and stable in exosomes: A promising biomarker for cancer diagnosis. Cell Res 25: 981-984, 2015 .

36. Weng W, Wei Q, Toden S, Yoshida K, Nagasaka T, Fujiwara T, Cai S, Qin H, Ma Y and Goel A: Circular RNA ciRS-7-A promising prognostic biomarker and a potential therapeutic target in colorectal cancer. Clin Cancer Res 23: 3918-3928, 2017.

37. Yang X, Xiong Q, Wu Y, Li S and Ge F: Quantitative proteomics reveals the regulatory networks of circular RNA CDR1as in hepatocellular carcinoma cells. J Proteome Res 16: 3891-3902, 2017.

38. Zheng Q, Bao C, Guo W, Li S, Chen J, Chen B, Luo Y, Lyu D, Li Y, Shi G, et al: Circular RNA profiling reveals an abundant circHIPK 3 that regulates cell growth by sponging multiple miRNAs. Nat Commun 7: 11215, 2016.

39. Kulcheski FR, Christoff AP and Margis R: Circular RNAs are miRNA sponges and can be used as a new class of biomarker. J Biotechnol 238: 42-51, 2016.

This work is licensed under a Creative Commons Attribution-NonCommercial-NoDerivatives 4.0 International (CC BY-NC-ND 4.0) License. 